DE

M E D I C I N A

T R O P I C A L

$\mathrm{DE}$

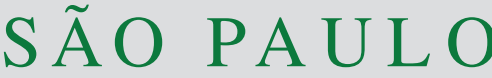

JOURNAL OF THE SÃO PAULO INSTITUTE OF TROPICAL MEDICINE

1Universidade de São Paulo, Instituto de Medicina Tropical de São Paulo, Laboratório de Protozoologia, São Paulo, São Paulo, Brazil

Correspondence to: Heitor Franco de Andrade Junior

Universidade de São Paulo, Instituto de Medicina Tropical de São Paulo, Laboratório de Protozoologia, Av. Dr. Enéas de Carvalho Aguiar, 470, $1^{\circ}$ andar, CEP 05403-000,

São Paulo, SP, Brazil

Tel +55 1130617010

E-mail: hfandrad@usp.br

Received: 10 July 2019

Accepted: 14 August 2019

\section{Saliva collection and detection of anti-T. gondii antibodies of low-income school-age children as a learning strategy on hygiene, prevention and transmission of toxoplasmosis}

Miriam de Souza Macre', Luciana Regina Meireles', Barbara Fialho Carvalho Sampaio', Heitor Franco de Andrade Júnior ${ }^{(1)} 1$

\section{ABSTRACT}

School-age children are a social group in which blood collection for laboratory testing can be perceived as an invasive procedure, with low acceptance and tolerance of stakeholders. This problem could be circumvented by replacing serum samples with saliva. For this purpose, and to make the collection of saliva samples playful and instructive for children, educational activities on hygiene and toxoplasmosis transmission and prevention were performed using toys and audiovisual tools. The target audience consisted of 7-10 year-old children from low-income families who attended public schools in the city of São Paulo. Saliva samples were used in a previously described in-house Enzyme-Linked Immunosorbent Assays (ELISA) to detect anti-Toxoplasma gondii IgG antibodies and establish the immunological status of each of the participants. One year later, children's memory and fixation of concepts regarding hygiene habits, as well as transmission and prevention of toxoplasmosis were tested in the same schools, by means of a questionnaire application, using students who did not participate in the first intervention as controls. The prevalence of positive anti-T. gondii IgG among students was 50\% (82/164). One year later, 45 children had more knowledge on toxoplasmosis (28/45 vs 29/147) and they drew the cat's involvement in the transmission of toxoplasmosis more often than controls (28/45 vs 29/147). Sorted according to the presence of specific IgG in saliva, recovered positive students presented worse memory of the above cited knowledge as did saliva-negative IgG students, but both groups had isolated higher frequency of fixed knowledge than non-intervened students. Our data show that there is a high prevalence of $T$. gondii infection in school-children from low-income areas; saliva is an alternative to blood for anti-T. gondii IgG detection; and a one-day educational intervention in school-children was effective in promoting knowledge fixation on hygiene and toxoplasmosis transmission and prevention after one year.

KEYWORDS: Toxoplasma gondii. Children. Memory. Saliva. IgG. Learning. Toxoplasmosis. School-age.

\section{INTRODUCTION}

Toxoplasmosis is a complex parasitic infection transmitted with high frequency in childhood, usually asymptomatic and laboratory diagnosis is performed by specific IgG anti-Toxoplasma gondii detection in serological assays. Toxoplasmosis is acquired either by oocyst ingestion due to poor hygiene habits in poor sanitized areas or by ingestion of cyst containing meat ${ }^{1}$. The disease has also been involved in behavior disorders and learning inability, as detected in human and experimental models ${ }^{2}$. Incidence studies are especially difficult in protected populations such as 
children. Serological studies in adult populations generally show a slight increase in annual prevalences, so that children are at higher risk of infection than adults. The prevalence of serologically positive people increases in adolescence, reducing the risk of contracting the infection, explaining the high proportion of young women of childbearing age with IgG anti-T. gondii ${ }^{3}$. Toxoplasmosis incidence in this age-group is higher probably due to poor sanitation, environmental exposure and inadequate hygiene habits. The prevention of diseases with oral or oral-fecal transmission as toxoplasmosis through the improvement of hygiene habits needs to be implemented before the first cycle of the Elementary School.

Today, kindergarten and pre-school teachers are responsible for teaching most hygiene concepts for children, bearing in mind that most mothers have jobs and spend less time with their children. This is a challenging task for teachers in crowded classrooms. New interactive interventions could help the teaching of specific preventive measures not to acquire toxoplasmosis, but the effective evaluation of these interventions is difficult because answering to the questionnaires require that children understood and fixed the information and remember the teachings, aside from they other bias ${ }^{4}$.

Any actions towards children are complex and fraught with prejudices, probably due to the decrement in the number of children per couple, leading to greater parental concerns. These concerns reduce public health interventions in these groups, protected by both, society and parents ${ }^{5}$. Invasive procedures should be avoided in this age-group, such as blood sampling, representing a huge obstacle for the investigation of chronic infections that are usually made by serology, as in the case of toxoplasmosis. To circumvent this obstacle, saliva is a fluid material that contains a small proportion of serum, exuded from the mucosa or the crevicular fluid from the teeth- gingiva border and could be an alternative source for the detection of specific IgG in conventional tests ${ }^{6}$. Saliva samples collection would be more acceptable for parents of this age-group children, who would in turn be more cooperative for the saliva collection.

Public schools in São Paulo Metropolitan area offer an opportunity to test educational interventions and to evaluate the prevalence of Toxoplasma infection through specific IgG detection. Children's approach one year later would allow to study the memory and fixation of concepts and interventions related to the transmission and prevention of toxoplasmosis. In the present study, we aimed to investigate the prevalence of anti-Toxoplasma IgG in São Paulo low-income school-age children, through the search of specific IgG in saliva, collected after a one-day educational intervention on hygiene habits and measures to avoid transmission and prevent toxoplasmosis in the school. The effect on memory fixation and learning on Toxoplasma infection, as well as on the hygiene teaching intervention was tested by questionnaires on the remaining students one year after the intervention, using students of the same school that did not participate in the intervention as controls.

\section{MATERIALS AND METHODS}

\section{Sample}

All students from the $3^{\text {rd }}$ and $4^{\text {th }}$ Brazilian grades, 7-10 years old, from a large Public School in a lowincome neighborhood from São Paulo Metropolitan area were invited to participate in the study, during the spring of 2003. Parents or guardians were informed and consented with the student participation in both stages of the research. The children also consented to participate in the study. During the one-day intervention, with cooperation of teachers and school personnel, 164 saliva samples were collected and identified. After one year, the same school was visited and 192 students from the $4^{\text {th }}$ and $5^{\text {th }}$ grades completed a simple questionnaire approaching the transmission of toxoplasmosis by contact with food, meat, the environment and pet animals, such as cats, dogs or both. Knowledge on the disease was evaluated as a qualitative knowledge when any of the toxoplasma transmission questions was correctly answered. Moreover, a knowledge qualitative index, defined as the sum of correct questions, subtracting wrong questions, such as dog transmission, was also evaluated. After the survey, 45 questionnaires of students who had participated in the previous intervention and had saliva samples tested for the presence or absence of anti-T. gondii IgG in saliva were found. We have also collected blood and saliva from 17 healthy school-age volunteers for use as controls, with pairing by age and gender, as elsewhere described, after the application of consent form ${ }^{6}$. The institutional Research and Ethics Committee (CAPPESQ) approved the study under the protocol $\mathrm{N}^{\circ} 365 / 00$.

\section{Educational intervention and saliva sampling}

First, students attended a 30-minute class given by a trained teacher in a large classroom. After this, all students received printed material consisting of various folders and printed games, as well as drawing material. The master booklet, prepared by the Protozoology Laboratory of the Tropical Medicine Institute of São Paulo, was a simplified 
teaching vehicle for elementary students, their families and teachers. The content is composed of illustrated educational material, avoiding the use of long texts. This booklet facilitates the guidance and coordination of the class held at school, and had been previously distributed to students and their families, including the guide for understanding the informed consent. The booklet also contained short and practical information, the purpose of which was for communication to reach the entire family group, children and parents.

The practical-educational activities were applied to groups of 30-40 students from the $3^{\text {rd }}$ and $4^{\text {th }}$ grades, without distinction from those whose parents authorized or not to participate in the research. The intervention allowed the students to ask questions and answer provocative questions formulated to increase the children's participation. The material for drawings and games and other activities was distributed and encouraged the individual participation of each child, who was questioned on hygiene concepts and toxoplasmosis. After the classroom intervention, all students, regardless of parental consent, could voluntarily participate in saliva collection. The collection process was initiated with oral cavity hygiene with $25-40 \mathrm{~mL}$ of commercial mouthwash (Listerine ${ }^{\circledR}$ ), followed by three mouthwashes with drinking water. After the washes, the student spit the saliva into a $45 \mathrm{~mL}$ plastic tube until reaching an ideal volume of $5 \mathrm{~mL}$, whenever possible. Tubes containing saliva from students with signed informed consent were identified and stored in an ice bath until transport and processing at the Protozoology Laboratory on the same day. Students without signed informed consent could participate voluntarily in the activities, but their saliva samples were discarded.

\section{One-year follow-up}

One year after the intervention, the students' fixed memory of the educational intervention was assessed. To this end, the teachers applied a simple and short questionnaire with drawings in various classrooms. These classes had many students who participated in the previous year's intervention and others who did not participate and were considered control students. The test contained 10 multiple-choice illustrated tests on the chain of transmission of zoonoses, with particular emphasis on toxoplasmosis. In addition, there were open areas for drawing animals involved in the Toxoplasma cycle. Each file was clearly identified and sent to IMTSP, where students were selected according to their participation in the previous intervention one year before and also to the results of specific anti-T gondii $\operatorname{IgG}$ in saliva. No previous educational intervention was performed before the test was applied, but after that, a new presentation highlighted for all students the importance of hygiene and zoonosis prevention.

\section{Saliva processing for $\lg G$ assay}

Briefly, saline borate buffer $(\mathrm{NaCl} 0.1 \mathrm{M}$, Borate buffer $0.05 \mathrm{M}, \mathrm{pH} 7.2$ ) was added to $2-5 \mathrm{~mL}$ of saliva samples to complete $7.5 \mathrm{~mL}$; the solutions were vortexed and centrifuged at $4{ }^{\circ} \mathrm{C}$ at $1,000 \mathrm{xg}$ for $15 \mathrm{~min}$. The supernatant was collected; ethanol absolute $\mathrm{v} / \mathrm{v}$ was added to $1 \mathrm{~mL}$ aliquots in conic tubes, vortexed and stored at $-12{ }^{\circ} \mathrm{C}$ by at least one hour. The tube was centrifuged at $4{ }^{\circ} \mathrm{C}$ at $10,000 \mathrm{x}$ g for $5 \mathrm{~min}$, the supernatant was discarded, and the pellet was re-suspended in $0.2 \mathrm{~mL}$ of saline borate buffer, resulting in $5 \mathrm{x}$ concentrated saliva IgG. For the standardization of tests, we used saliva from 17 adult volunteers with known serology, processed in the same way as school-children saliva.

\section{ELISA anti-T. gondii IgG assays}

These assays are routinely performed and were reported elsewhere ${ }^{7}$. Briefly, 96-well polystyrene plates were adsorbed with soluble $T$. gondii saline extract from tachyzoites $(1 \mu \mathrm{g} / \mathrm{mL})$ and free-binding sites were blocked with PBS containing $0.02 \%$ Tween 20 and $1 \%$ skimmed milk (PBSTL). The plate was washed with PBSTL four times between steps. Sample (saliva $5 x$ or 1:100 serum dilution) was incubated at $37^{\circ} \mathrm{C}$ for one hour. Anti-human $\mathrm{IgG}$ peroxidase conjugate was added and again incubated for one hour at $37^{\circ} \mathrm{C}$. Bound conjugate was detected by TMB commercial solution for $30 \mathrm{~min}$ in a dark chamber. The reaction was stopped by adding $25 \mu \mathrm{L}$ of $\mathrm{HCl} 4 \mathrm{~N}$, and the $450 \mathrm{~nm}$ optical density (O.D.) of each well was determined in an automatic microplate reader (Labsystem Multiskan M.S., Labsystem, Vantaa, Finland). The 99\% (mean plus 3 Standard Deviation - SD) threshold cut-off was determined using saliva from 11 volunteers with known negative serology to toxoplasmosis.

\section{Statistical analysis}

Questionnaires were included in a database together with $\mathrm{IgG}$ results of individual students. Non-participating students' questionnaire results were also included as controls. These populations were grouped by two-tailed Fisher's exact test or Chi-square with 90\% test force and analyzed using the GraphPad Prism contingency tests. Data were considered statistically significant when the likelihood of equality was lower than $5 \%(\mathrm{p}<0.05)$. 


\section{RESULTS}

\section{Saliva anti-T. gondii IgG ELISA}

The initial educational intervention was carried out as described in the Methods section. Six classes of schoolchildren from the $3^{\text {rd }}$ and $4^{\text {th }}$ grades resulted in a collection of 164 saliva samples for the anti-T. gondii IgG detection by ELISA and 164 student's drawings to analyze the initial impact of the intervention.

Using the saliva anti-T. gondii IgG to define the contact with the parasite of toxoplasmosis, it was possible to identify a positive frequency in this population of $50 \%$ (82/164), with individual distributions shown in Figure 1. The value threshold or cut-off was calculated using saliva from seronegative volunteers using the $99 \%$ threshold (mean plus $3 \mathrm{SD}$ ) which is a more restrictive definition.

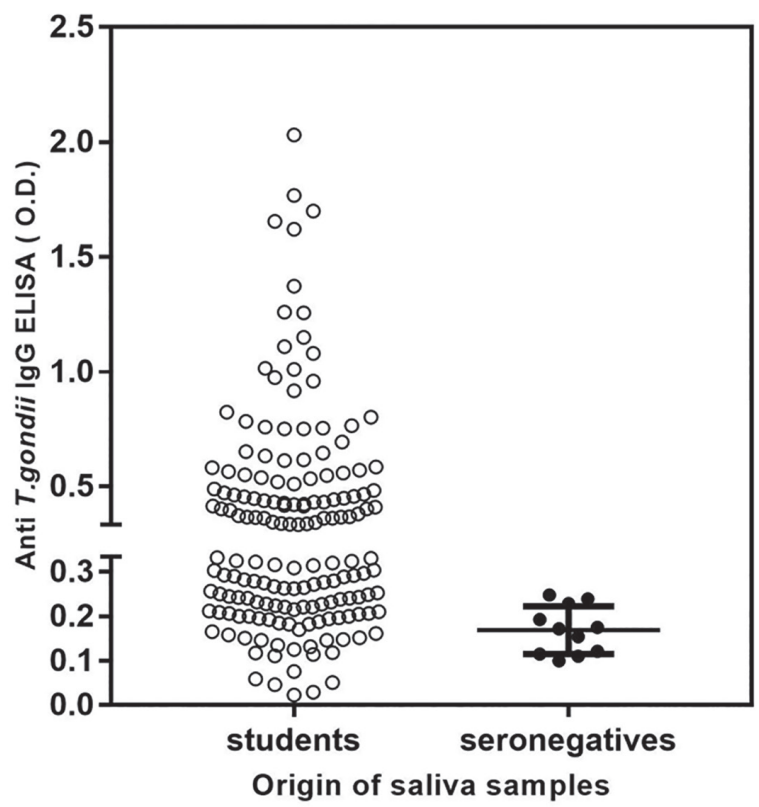

Figure 1 - Distribution of ELISA results obtained in saliva of students undergoing the intervention. The $Y$ axis was interrupted in the $99 \%$ cut off threshold calculated using the saliva reactivity of volunteers with known negative serology to T. gondii.

\section{Intensive learning activity effect on students' memory on hygiene and the zoonosis}

The material produced in class by these students during intervention was collected and evaluated. It was found that most children reported the cat's presence in spontaneous drawings $(74 \%, 121 / 164)$ immediately after the intervention. After one year, children from the $4^{\text {th }}$ and $5^{\text {th }}$ grades of the same school answered a questionnaire described in Methods. We compared the students subjected to the intervention with their counterparts that did not participate. For literacy, there are similar proportions in the exposed students (45\%) and the unexposed group (57\%). There are also no differences between groups according to the socio-economic criteria, such as household appliances (televisions, computers, refrigerators) and numbers of compartments in the residence.

The proportion of any knowledge regarding the toxoplasmosis transmission was higher $(\mathrm{P}<0.001)$ in the exposed group (39/45) as compared to the unexposed ones (41/147). Transmission was associated with animals (exposed 28/45, unexposed 29/147, $\mathrm{p}<0.001$ ), however with no differences when pets, dogs or cats were defined. Quantitative knowledge has also been evaluated, attempting to define a mean knowledge based on correct and wrong questions. In this analysis, exposed students presented a higher mean index (2.46 \pm 0.97$)$ when compared to unexposed ones $(2.06 \pm 1.11)$ and higher frequency of correct answers $(\mathrm{p}<0.05)$ as compared by frequency of individual value categories by the Chi-square statistics. These data show that one day educational intervention induces a specific memory of knowledge after one year. We recovered the presence of anti-T. gondii $\mathrm{IgG}$ in these 45 students, from whom 24 presented antibodies in saliva ( $\operatorname{IgG}+$ group) while 21 had no specific antibodies in saliva (IgG - group) and they had the memory indices described above. These groups were compared by the frequency of any knowledge on the sources of infection of toxoplasmosis, showing that $\operatorname{IgG}+$ students $(18 / 24)$ had significant $(\mathrm{p}<0.05)$ less memories on T. gondii transmission as compared to their IgG- colleagues (21/21). Looking for any knowledge on the transmission of toxoplasmosis, each isolated exposed group had more knowledge than non-exposed students ( $\operatorname{IgG}+$ students, $18 / 24$ vs $41 / 147, p<0.001$; IgG- students $21 / 21$ vs $41 / 147$, $\mathrm{p}<0.001$, Fisher exact test). Quantitative mean indices were similar between exposed groups, and higher for each one of them when compared to non-exposed students $(\mathrm{p}<0.05)$. These aspects were suggestive of some interference regarding the memory on toxoplasmosis infection one year after the intervention, but the results were only suggestive of the interference in the process of memory on toxoplasmosis, as exposition to intervention made the students retain some memory on toxoplasmosis transmission after one year.

\section{DISCUSSION}

Our data showed that it is possible to detect specific anti- $T$. gondii $\mathrm{IgG}$ using saliva as an alternative source of IgG in school-children from public schools of Sao Paulo, aged 7 to 10 years old.

Saliva as a source of $\operatorname{IgG}$ are frequently associated with false-negative ELISA results when compared to 
serum samples due to low levels of $\operatorname{IgG}$ in the absence of purification and concentration strategies, but rarely to false-positive results, thus its positivity is reliable. There are several reports on saliva false-negative results but very few on false-positive ones, clearly detailed in a recent report in which IgG subclasses were also studied ${ }^{8}$. Obviously, when saliva in used, a careful analysis must be performed, or the use of more adequate tests as Protein A capture assays in concentrated purified samples ${ }^{6}$, but these tests were not available at the time of the present study. Using washed and concentrated saliva, we demonstrated a $50 \%$ specific $\mathrm{IgG}$ positivity, probably related to the lack of hygiene habits and low local sanitary conditions.

The prevalence of toxoplasmosis in this age group is high, as expected, because of the evolution of the prevalence of seropositivity in pregnant women in the same area ${ }^{3}$. The progression of prevalence in adults is lower than the ones in young ages, thus these mothers must had acquired the agent most likely before childbearing age, as a consequence of a high exposure in childhood due a lack of adequate hygiene habits. Seronegative pregnant women of similar background areas had low seroconversion rate/year, a fact corroborated by the low incidence of diagnosed congenital toxoplasmosis ${ }^{3}$. This higher prevalence in children is related to the health and socioeconomic conditions in peripheral areas of big cities, while the incidence of $T$. gondii infection is much lower in high-income university students ${ }^{7}$. The transmission of toxoplasmosis in this population should be related to oocyst ingestion, as reported in a similar population ${ }^{9}$, and also to the higher environmental exposure of low-income children, as described in African populations ${ }^{10}$.

Children exposed to one-day intensive interaction showed a great immediate fixation of knowledge, with most students drawing the correct animals as Toxoplasma hosts and also the preventive concepts. For the assessment of knowledge fixation after one year, students have also shown knowledge fixation, supporting this type of interaction for training on hygiene issues. Proving the effective impact of this type of intervention is difficult, as discussed in a recent meta-analysis ${ }^{11}$, but most studies confirmed some beneficial effect provided by this type of intervention. Our data show that some sort of memory remained after this intervention, which should not directly result in an effective benefit, as in a study on the prevention of dental plaques, others groups studied the learning capacity of Toxoplasma-infected children, showing a less effective learning especially in mathematics, but this study had sampling problem, having analyzed a restrict number of infected children ${ }^{12}$. Another study in Poland showed a similar school performance of Toxoplasma-infected or non-infected children, finding only a gender difference ${ }^{13}$. This learning effect is difficult to analyze in low-income populations, submitted to different expositions and conditions, in children with less parental care and high exposition to environmental sources of infection.

We found a significant difference in knowledge fixation when we tested children with toxoplasmosis after one year, a worse knowledge fixation when compared to non-infected counterparts. Despite the adequacy of the sample, this finding may reflect only a cluster of poor conditions in the infected population, that may have worse parental care or social conditions that may result in poorer knowledge fixation due to causes other than toxoplasmosis. In this context, the selection bias may have occurred as the relative loss of attention may affect the knowledge fixation, regardless of the intelligence coefficient of the child. Despite the controversy, some studies reported differences in cognitive aspects and memory problems in children previously infected with T. gondii $^{14}$. These studies are difficult to be unbiased, as a worse performance due to behavioral and social changes that also increase exposure to $T$. gondii infection could also affect the school performance. The infection could act only as a marker of other risks, with no causal relationship between the student performance and chronic toxoplasmosis, a fact that has been reported elsewhere ${ }^{15}$.

When carefully collected, purified and concentrated, saliva is a good alternative to blood for detection of anti T. gondii antibodies, avoiding invasive needle sampling in this age-group. We found a prevalence of $50 \%$ of $T$. gondii infection in low-income schoolchildren (7-10 years old) from Sao Paulo. These numbers reinforces the importance of hygiene teaching in elementary school that must be performed by both, teachers and specialized teams during specific, intense and focused one-day interventions, that we have proven to be effective in promoting knowledge fixation.

\section{ACKNOWLEDGMENTS}

We thanks the technical assistance of Roselaine P.A. Cardoso. HFA Jr. is a fellow of CNPq and FFM. We also thanks the Secretaria de Estado da Educação de São Paulo (Coordenação SP Zona Leste and staff of E.E. Antonio de Pádua Vieira) and the students that happily participated, supplying material for this work. This work was partially supported by LIMHCFMUSP-49.

\section{REFERENCES}

1. Barry MA, Weatherhead JE, Hotez PJ, Woc-Colburn L. Childhood parasitic infections endemic to the United States. Pediatr Clin North Am. 2013;60:471-85. 
2. Sinai AP, Watts EA, Dhara A, Murphy RD, Gentry MS, Patwardhan A. Reexamining chronic Toxoplasma gondii infection: surprising activity for a "dormant" parasite. Curr Clin Microbiol Rep. 2016;3:175-85.

3. Guimarães AC, Kawarabayashi M, Borges MM, Tolezano JE, Andrade Júnior HF. Regional variation in toxoplasmosis seronegativity in the São Paulo metropolitan region. Rev Inst Med Trop Sao Paulo. 1993;35:479-83.

4. Moore GF, Tapper K, Moore L, Murphy S. Cognitive, behavioral, and social factors are associated with bias in dietary questionnaire self-reports by schoolchildren aged 9 to 11 years. J Am Diet Assoc. 2008;108:1865-73.

5. Radisic G, Chapman J, Flight I, Wilson C. Factors associated with parents' attitudes to the HPV vaccination of their adolescent sons: a systematic review. Prev Med. 2017;95:26-37.

6. Sampaio BF, Macre MS, Meireles LR, Andrade Júnior HF. Saliva as a source of anti-Toxoplasma gondii IgG for enzyme immunoassay in human samples. Clin Microbiol Infect. 2014;20:072-4

7. Rodrigues JP, Frei F, Navarro IT, Silva LP, Marcelino MY, Andrade Júnior HF, et al. Seroepidemiological analysis of toxoplasmosis in college students. J Venom Anim Toxins Incl Trop Dis. 2015;21:1.

8. Cañedo-Solares I, Gómez-Chávez F, Luna-Pastén H, Ortiz-Alegría LB, Flores-García Y, Figueroa-Damián R, et al. What do antiToxoplasma gondii IgA and IgG subclasses in human saliva indicate? Parasite Immunol. 2018;40:e12526.

9. Dattoli VC, Veiga RV, Cunha SS, Pontes-de-Carvalho L, Barreto ML, Alcantara-Neves NM. Oocyst ingestion as an important transmission route of Toxoplasma gondii in Brazilian urban children. J Parasitol. 2011;97:1080-4.
10. Fan CK, Lee LW, Liao CW, Huang YC, Lee YL, Chang YT, et al. Toxoplasma gondii infection: relationship between seroprevalence and risk factors among primary schoolchildren in the capital areas of Democratic Republic of São Tomé and Príncipe, West Africa. Parasit Vectors. 2012;5:141.

11. Stein C, Santos NM, Hilgert JB, Hugo FN. Effectiveness of oral health education on oral hygiene and dental caries in schoolchildren: systematic review and meta-analysis. Community Dent Oral Epidemiol. 2018;46:30-7

12. Ferreira EC, Marchioro AA, Guedes TA, Mota DC, Guilherme AL, de Araújo SM. Association between seropositivity for Toxoplasma gondii, scholastic development of children and risk factors for T. gondii infection. Trans R Soc Trop Med Hyg. 2013;107:390-6.

13. Mizgajska-Wiktor H, Jarosz W, Andrzejewska I, Krzykała M, Janowski J, Kozłowska M. Differences in some developmental features between Toxoplasma gondii-seropositive and seronegative school children. Folia Parasitol (Praha). 2013;60:416-24.

14. Mendy A, Vieira ER, Albatineh AN, Gasana J. Toxoplasma gondii seropositivity and cognitive functions in school-aged children. Parasitology. 2015;142:1221-7.

15. Sugden K, Moffitt TE, Pinto L, Poulton R, Williams BS, Caspi A. Is Toxoplasma gondii infection related to brain and behavior impairments in humans? Evidence from a populationrepresentative birth cohort. PLoS One. 2016;11:e0148435. 